\title{
The role of IncRNA CASC2 on prognosis of malignant tumors: a meta-analysis and bioinformatics
}

\author{
Lingling $\mathrm{Yu}^{\prime}$ \\ Shengsong Chen ${ }^{2}$ \\ Hui Bao' \\ Weifang Zhang ${ }^{3}$ \\ Minqi Liao' \\ Qian Liang ${ }^{4}$ \\ Xiaoshu Cheng'
}

'Department of Cardiovascular Medicine, The Second Affiliated Hospital of Nanchang University, Nanchang of Jiangxi, 330006, China; ${ }^{2}$ Department of Respiratory and Critical Care Medicine, Jiangxi Provincial People's Hospital, Nanchang of Jiangxi, 330006, China; ${ }^{3}$ Department of Pharmacy, the Second Affiliated Hospital of Nanchang University, Nanchang of Jiangxi, 330006, China; ${ }^{4}$ Key Laboratory of Molecular Biology in Jiangxi Province, The Second Affiliated Hospital of Nanchang University, Nanchang of Jiangxi, 330006, China
Correspondence: Qian Liang

Key Laboratory of Molecular Biology in Jiangxi Province, The Second Affiliated Hospital of Nanchang University, No I Minde Road, Nanchang of Jiangxi 330006, China

Tel +86 (0)79l 86297276

Email fishuang@I26.com

Xiaoshu Cheng

Department of Cardiovascular Medicine, The Second Affiliated Hospital of Nanchang University, No I Minde Road, Nanchang of Jiangxi 330006, China Email xiaoshumenfan I26@I63.com
This article was published in the following Dove Press journal: OncoTargets and Therapy

Background: Cancer susceptibility candidate 2 (CASC2) is characterized as a tumor suppressor, which was first identified to be downregulated in endometrial carcinoma. Accumulating evidence was provided to testify the function of CASC 2 in malignant tumors. However, a systematic and quantitative assessment is not available. The present study was designed to evaluate the role of CASC2 in multiple carcinomas through meta-analysis and bioinformatics.

Materials and methods: A systematic assessment of the relationship of CASC2 with tumors was performed by using several computerized databases from inception to December 1, 2017. Pooled HR with $95 \%$ CI was calculated to summarize the effect. The data on prognosis of malignant tumors were also downloaded from The Cancer Genome Atlas (TCGA) project, OncoLnc, TANRIC and lncRNAtor database.

Results: A total of 13 studies with 966 cancer patients were pooled in the analysis to evaluate the prognostic value of CASC2 in multiple tumors and the clinical features. The results of the meta-analysis revealed that low expression levels of CASC2 were associated with poor overall survival (OS) (pooled HR=0.39, 95\% CI: 0.28-0.53, $P<0.0001$ ). CASC2 obviously has a negative correlation with advanced tumor node metastasis (TNM) stage, lymph node metastasis $(\mathrm{LNM})$ and $\mathrm{T}$ stage, respectively $(P<0.05)$. There was, however, no significant difference in gender, distant metastasis and high differentiation $(P>0.05)$. In the Kaplan-Meier curves with log-rank analysis, higher expression of CASC2 was positively correlated with longer survival time than patients with a lower level $(P<0.05)$, including kidney renal clear cell carcinoma, brain lower grade glioma, pancreatic adenocarcinoma and sarcoma.

Conclusion: Findings from this meta-analysis suggest that lower expression of CASC2 is associated with poorer prognosis of cancers, as well as advanced TNM, LNM and T stage. Data from the bioinformatics analysis revealed that higher expression of CASC2 was related to longer OS in patients with malignant tumors.

Keywords: CASC2, malignant tumors, prognosis, meta-analysis, bioinformatics

\section{Introduction}

There is ample epidemiological evidence indicating malignant tumors as the leading cause of mortality and morbidity worldwide, with $\sim 8.2$ million people dying from cancers and 14.1 million people being diagnosed with cancer every year according to the "global cancer report 2012" released by the World Health Organization (WHO). ${ }^{1-3}$ And worst of all, the number of new cases is expected to rise by about $70 \%$ over the next two decades. ${ }^{4}$ The latest research from the American Cancer Society showed that $\sim 1.7$ million new cancer cases and 600,000 cancer deaths were expected to occur in the USA in 2017..$^{5}$ Despite advances in the level of health care, oncotherapy is 
still confronted with great challenges due to the delayed diagnosis, poor prognosis, recurrence or metastasis, as well as cancer associated mutation. ${ }^{6,7}$ Tumor molecular markers, a class of chemical substances that specifically reflect the existence and growth of malignant tumors, can effectively and greatly evaluate tumor screening, diagnosis, prognosis, and recrudescence and, to date, have been widely used in clinical treatment of tumors. ${ }^{8}$ Therefore, analysis and measurement of the specific biomarkers in malignant tumors, specifically the connection between the expression levels of biomarkers and tumor progression, may provide insights into opportunities and priorities for prevention, research, and clinical treatment of tumors.

Long non-coding RNAs (lncRNAs) are defined as a group of nonprotein-coding RNAs, but interacting with proteins, with a length of $>200$ nucleotides that can regulate gene expression at the transcriptional or posttranscriptional level. ${ }^{9}, 10$ Accumulating evidence suggests that lncRNA played an important role in various cellular biological processes, including cell growth, cell cycle, proliferation, differentiation, apoptosis, metabolism, and carcinogenesis. ${ }^{1-16}$ Recently, human genomic sequence data have shown that more than $90 \%$ of the human genomic sequences are transcribed to produce lncRNAs. ${ }^{17-19}$ Furthermore, studies have demonstrated that there are $18 \%$ of lncRNAs associating with human tumors, obviously higher than those of $9 \%$ of human protein-coding genes which only account for $1 \%$ of the total genomic sequences. ${ }^{20,21}$ All these suggested that lncRNAs might act as major contributors to carcinogenesis and cancer progression and the dysregulated lncRNAs in most cancers may be used as markers for cancer diagnosis and prognosis, as well as potential therapeutic targets.

The lncRNA cancer susceptibility candidate 2 (CASC2), which is located on chromosome 10q26, is a novel lncRNA initially identified to be downregulated in endometrial cancer and has been characterized as a tumor suppressor. ${ }^{22,23}$ An increasing number of studies have shown that CASC2 is abnormally expressed in various malignancies and plays a tumor-suppressive role through inhibiting cell proliferation, migration, invasion, and inducing apoptosis, such as glioma, gastric cancer, nonsmall-cell lung cancer, colorectal cancer, renal cell carcinoma, and endometrial cancer. ${ }^{24-37}$ These results have provided new insights into the role of CASC2 in the development of cancers and indicated the potential application of CASC2 as a prognostic marker and therapeutic target. To date, however, there are few reports concerning the relationship between the novel IncRNA, CASC2, by functioning as a tumor suppressor in various cancers and patient outcomes. Furthermore, there were limitations in most individual studies assessing the implication of CASC2 levels in cancer due to small sample sizes and discrete outcome. And, to the best of our knowledge, there has not been a systematic and quantitative assessment of the published studies available. Therefore, in the present study, we performed a correlation analysis based on metaanalysis and bioinformatics methods to explore the relationship between the expression of CASC2 and the prognosis of malignant tumors.

\section{Materials and methods}

\section{Literature search and study design}

A meta-analysis was conducted and reported according to standard guidelines of the meta-analysis of original studies. ${ }^{38,39}$ We performed a systematic search of published studies before December 1, 2017 in PubMed, Embase, Web of Science and Cochrane Library by using the following medical subject headings and terms without restrictions: ("long noncoding RNA" OR "Inc RNA" OR "cancer susceptibility candidate 2" OR “CASC2") AND (“cancer" OR "tumor" OR "neoplasm" OR “carcinoma") AND ("survival" OR "prognosis" OR "prognostic" OR "metastasis"). Searching was done without restriction on publication years. The strategy was adjusted for each database to maximize the chances of finding the appropriate articles. In addition, we reviewed the reference lists of retrieved articles to identify any studies that were not identified from the preliminary literature searches.

\section{Selection criteria for study inclusion}

Two researchers evaluated all of the included studies and extracted the data independently. Studies were included in the meta-analysis if they met the following predetermined inclusion criteria: 1) reported original data and published as a full peer-reviewed article; 2) all tumors had to be confirmed by clinical diagnosis; 3 ) the subjects in every study included cancer samples and normal controls; 4) published in the English language; 5) the relationship between CASC2 expression and OS, or pathological parameters such as lymph node metastasis (LNM), advanced tumor node metastasis (TNM) stage, or the difference of gender, with respect to CASC2 expression; 6) the level of CASC2 expression was measured in human tumor tissue by RT-PCR, and patients were grouped according to CASC2 level; 7) provided estimates of HR or OR with $95 \%$ CI.

The exclusion criteria were as follows: 1) cell or animal experiments, and letters, case reports, reviews, conference 
reports or commentaries; 2) the required data could not be extracted from the original article, the article was not found in full or had been published repeatedly; 3) only the latest publication was included if the same data subsets were reported; 4) only the single subunit of the CASC2 gene was studied.

\section{Data extraction}

Data extraction was conducted independently by two investigators from each retrieved article according to the selection standards. Decisions were made and any disagreements regarding decisions were resolved by discussion and consensus with a third reviewer. The following information was extracted from the studies: name of the first author, year of publication, country of origin, tumor type, sample size of cases, the number of patients with high difference (HD), LNM and distant metastasis (DM) in high or low expression of CASC2, HR and 95\% CI of CASC2 value for $\mathrm{OS}$, the reference gene and detection method of CASC2. Then, a database was established after the selected data were arranged and verified.

\section{Statistical analysis}

We pooled HR with 95\% CI from each study separately for each outcome using a fixed effects meta-analysis. ORs with $95 \%$ CIs were used to assess the associations between CASC2 expression and tumor parameters, including gender, TNM, LNM, DM, T stage and HD. A fixed or random effect model was applied depending on the heterogeneity analysis results in this meta-analysis, and the fixed effect model was used when a significant $\mathrm{Q}$ test with $P>0.05$ or $\mathrm{I}^{2}<50 \%$. Begg's funnel plots and Egger's was used to examine the underlying publication bias. All statistical analysis were done with Stata Software 12.0 version, $P<0.05$ was considered significant.

\section{Bioinformatics analysis}

The expression of CASC2 and its effect on the prognosis of malignant tumors were analyzed and downloaded from the Cancer Genome Atlas (TCGA) project, OncoLnc, TANRIC and lncRNAtor database (http://cancergenome.nih.gov/). Cox proportional hazards regression models were generated for OS of tumors, which incorporated multiple variables. CASC2 expression was then analyzed through the raw data and compared with the prognostic data of the patients with malignant tumors. Additionally, the prognostic significance of CASC2 was also analyzed using Kaplan-Meier plotter (http://kmplot.com).

\section{Results Included literatures}

We retrieved 62 articles from our preliminary search initially. After duplicate articles had been removed, 42 articles were left and these articles were then screened. Of these, 20 articles were excluded based on titles and abstracts that did not satisfy some or all of the inclusion criteria and 22 articles were identified for full review. Nine studies were then excluded due to lack of specific report on the association of CASC2 with malignant tumor or insufficient data in the original studies. Finally, the remaining 13 articles were included in the meta-analysis. The results of the literature search are shown in Figure 1.

\section{Characteristics of eligible studies}

The characteristics of the 13 articles are summarized in Table 1. Among these 13 studies, a total of 966 patients were represented, with a mean sample size of 74.3 (with a range from 47 to 133). All studies were conducted in China and were published between 2016 and 2017. There were 11 types of carcinomas in this meta-analysis, including hepatocellular carcinoma (HCC), ${ }^{29,35}$ glioma, ${ }^{30,31}$ nonsmall-cell lung cancer (NSCLC), ${ }^{25}$ colorectal cancer (CRC),${ }^{26}$ esophageal squamous cell carcinoma (ESCC), ${ }^{40}$ thyroid carcinoma (TC), ${ }^{34}$ pancreatic cancer (PC), ${ }^{32}$ lung adenocarcinoma (LAC), ${ }^{41}$ pituitary adenoma (PA), ${ }^{42}$ cervical cancer $(\mathrm{CC}),{ }^{28}$ and gastric cancer (GC) ${ }^{33}$ All these patients were divided into two groups (high and low expression of CASC2) according to the CASC2 measurement results by RT-PCR in cancerous specimens. All of the diagnoses of TNM, DM, LNM, T stage and HD were dependent on individual pathology. The reference gene of CASC2 in these studies was found to be inconsistent, including GAPDH, ${ }^{26,28-30,34,35,37}$ RNU6B, ${ }^{32}$ and $\beta$-actin. ${ }^{25,33}$ The Newcastle-Ottawa Scale confirmed that all studies were of good quality.

\section{Association between the CASC2 expression and OS}

The association between the CASC2 expression and overall survival (OS) is shown in Figure 2. We performed a cumulative meta-analysis to assess the function of CASC2 for OS in patients with cancers. There were 5 of 13 included studies with 995 patients reporting the relationship between OS and CASC2. The fixed effects model was used because no heterogeneity was found among those five studies (unadjusted $\mathrm{I}^{2}=34.2 \%, P=0.193$; adjusted $\mathrm{I}^{2}=0.00 \%, P=0.452$ ). There was a graded reduction in unadjusted event rates for OS from high to low expression of CASC2 (pooled $\mathrm{HR}=0.40$, 


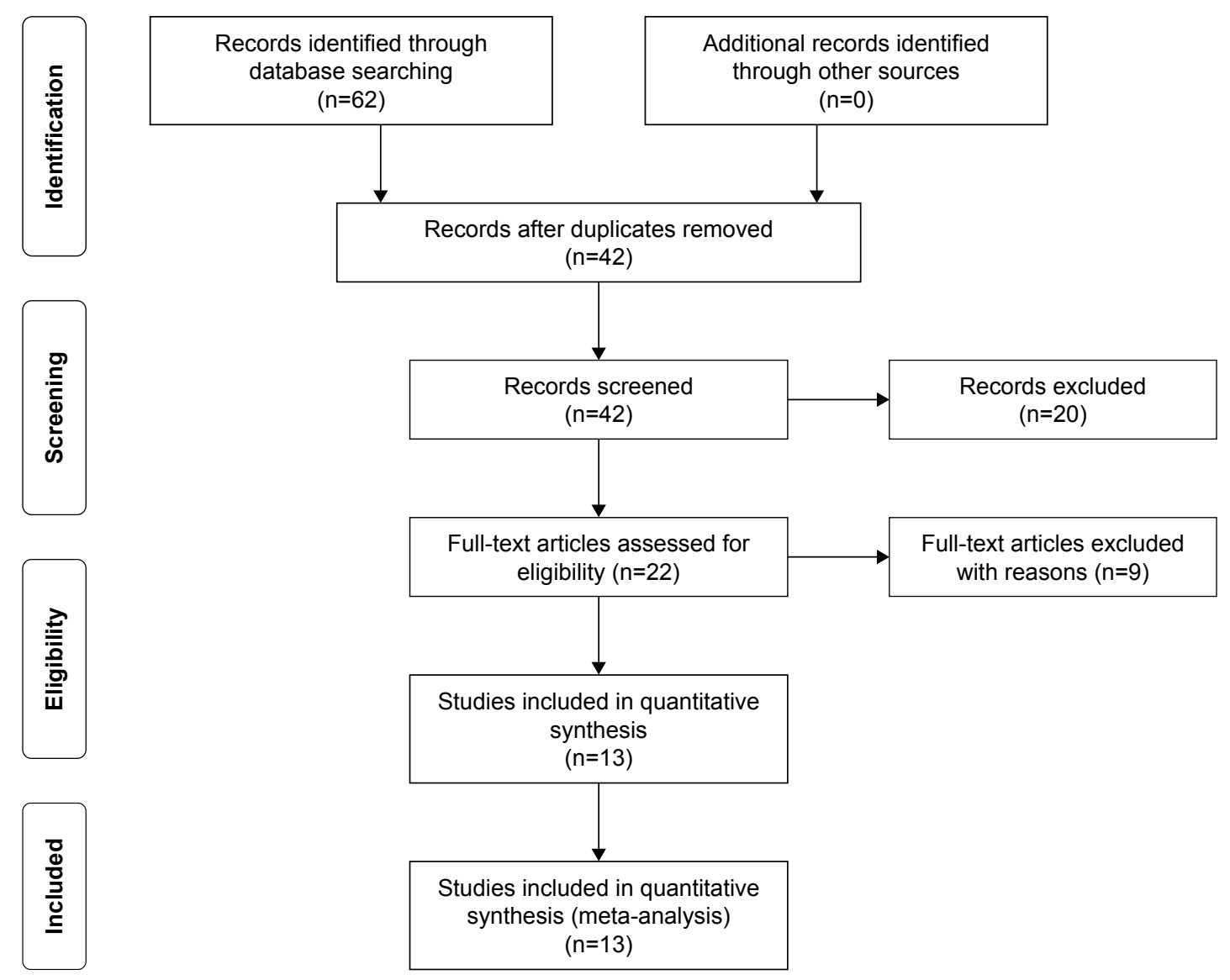

Figure I Flow diagram of the study search and selection process in the meta-analysis.

95\% CI: 0.29-0.54, $P<0.0001$ ) (Figure 2A). After further adjustment, the difference still existed that the OS of patients with lower expression of CASC2 was obviously decreased (pooled HR $=0.39$, 95\% CI: $0.28-0.53, P<0.0001$ ) (Figure 2B). These results from our meta-analysis study suggested that the expression of CASC2 was independently associated with OS among patients with cancers.

\section{Association between CASC2 expression and clinicopathological parameters of malignant tumors}

The correlations between CASC2 expression and cancers in gender, TNM, LNM, DM, T stage and HD are presented in Figure 3. There was no obvious heterogeneity in these studies, and the fixed effects model was used $\left(\mathrm{I}^{2}=0.0 \%, P=0.304\right.$; $\mathrm{I}^{2}=11.8 \%, P=0.332 ; \mathrm{I}^{2}=13.4 \%, P=0.327 ; \mathrm{I}^{2}=16.4 \%, P=0.309$; $\left.\mathrm{I}^{2}=00.0 \%, P=0.623 ; \mathrm{I}^{2}=00.0 \%, P=0.866\right)$. As it shown, we observed that there was no difference in CASC2 expression between the male and female patients with malignant tumors (pooled OR=0.86, 95\% CI: 0.65-1.14, $P>0.05$ ) (Figure 3A). People with TNM stage III-IV malignancies showed lower expression of CASC2, compared with those with stage I-II (pooled OR $=0.36,95 \% \mathrm{CI}: 0.26-0.48, P<0.001$ ) (Figure $3 \mathrm{~B})$. CASC2 expression was also negatively related to LNM of cancer and $\mathrm{T}$ stage in cancer patients (pooled $\mathrm{OR}=0.62,95 \%$ CI: 0.43-0.89, $P<0.01$; pooled OR=0.42, 95\% CI: 0.25-0.72, $P<0.01$ ) (Figure $3 \mathrm{C}$ and $\mathrm{E}$ ). However, no significant difference was observed between the two groups in the DM and HD incidence (pooled $\mathrm{OR}=0.59,95 \% \mathrm{CI}$ : 0.33-1.07, $P>0.05$; pooled $\mathrm{OR}=0.64,95 \% \mathrm{CI}: 0.41-1.00, P>0.05)$ (Figure 3D and F). These results indicated that low CASC2 expression in patients with malignant tumors may predict increased probability of DM, LNM and T stage.

\section{Publication bias}

The potential publication bias of the present meta-analysis was evaluated by funnel plot and Beggs indicator test in Figure 4. The results showed no evidence of publication bias was detected in the gender analysis with the expression of CASC2 $(P>0.05)$ as well as LNM $(P>0.05)$ (Figure 4A and C). Similar results occurred in the shape of the Begg's indicator test, which did not reveal any evidence of obvious 


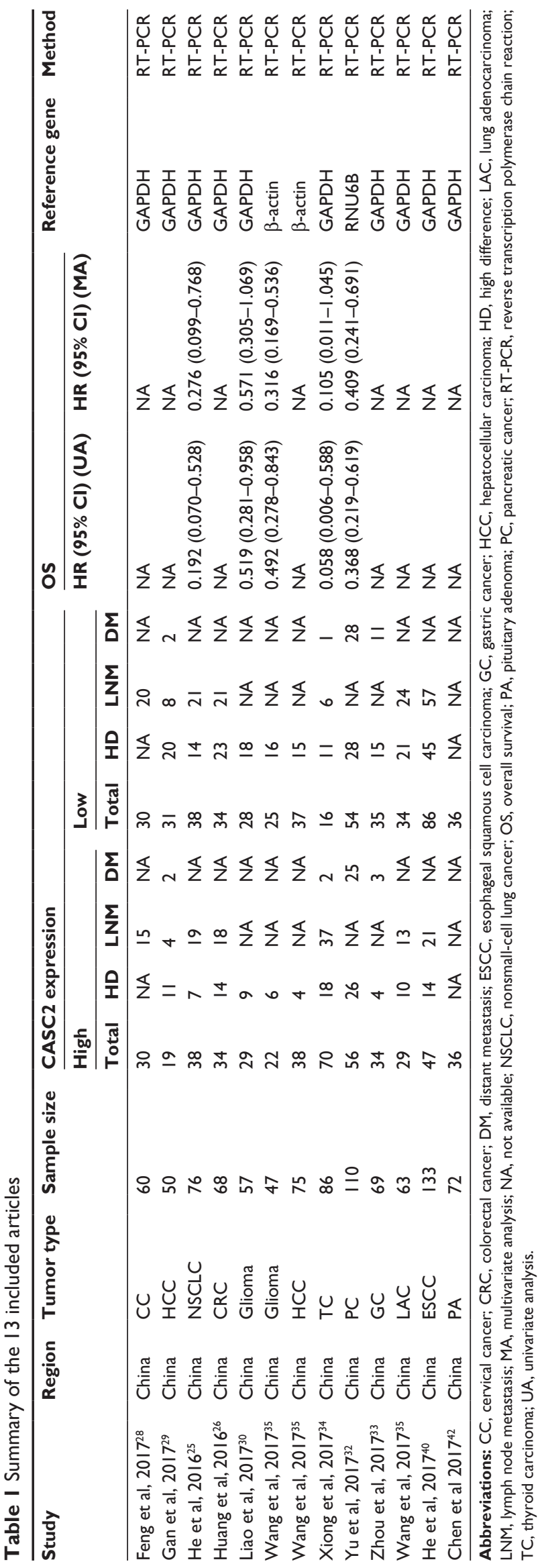

asymmetry (Figure 4D and F). For the analysis of TNM, but, there was certain publication bias in these 11 articles and the funnel plot and the Begg's indicator test revealed asymmetry $(P<0.05)$ (Figure 4B and $\mathrm{E}$ ).

\section{Sensitivity analysis}

The stability of the crude results was evaluated by sensitivity analysis in Figure 5. The results suggested that the conclusions are stable and robust because the pooled HR was not significantly affected by the exclusion of any single study.

\section{The prognostic significance of CASC2 expression in malignant tumors}

Bioinformatics analysis was performed to gain insight into the functional impact of the expression of CASC2 on cancers. As summarized in Figure 6, we performed multivariate analysis using Cox's hazard proportional model to evaluate whether CASC2 expression was an independent prognostic factor for malignant tumors, and four types of tumors were found with a significant difference, including KIRC, LGG, PAAD and SARC. Then, Kaplan-Meier curves with log-rank analysis were further used to explore the relationship between the expression of CASC2 and the OS rates of these patients in Figure 7. Similar to the results of our meta-analysis, we found that a higher CASC 2 expression was positively correlated with longer survival time than in patients with lower CASC2 expression $(P<0.05)$ (Figure 7A-D). These results suggested that CASC2 may be an independent predictor of tumors.

\section{Discussion}

Accumulating evidence has demonstrated that lncRNAs could play oncogenetic, tumor suppressive roles or both under different circumstances and in different tissues and cells, and they may promise to be the new tumor marker for diagnostic and prognosis of tumors. ${ }^{9,43} \mathrm{CASC} 2$, which was considered to be a tumor suppressor, has been reported by several original articles negatively relating to poor prognosis of cancers through its regulation of diverse cellular processes, including migration, invasion, proliferation, differentiation, and apoptosis. ${ }^{28,30,31}$ Our results from the meta-analysis and bioinformatics analysis also suggested that the lower expression of CASC 2 was consistently associated with poor prognosis of malignant tumors. The value of meta-analysis is illustrated by the fact that most studies individually lacked precision, a problem alleviated by pooling.

In our study, we found that sarcoma patients from 13 remaining articles presented poor OS, including HCC, 


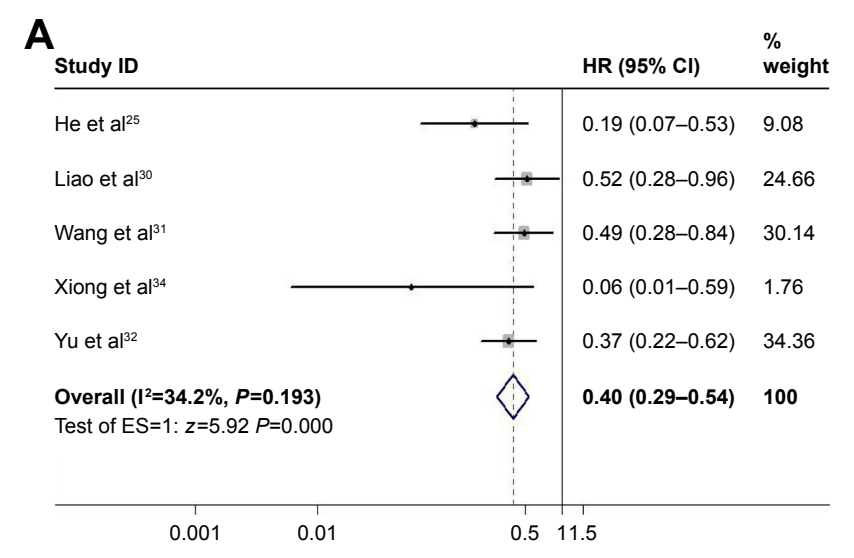

\begin{tabular}{|c|c|c|}
\hline $\mathbf{B}_{\text {Study ID }}$ & HR $(95 \% \mathrm{Cl})$ & $\begin{array}{l}\% \\
\text { weight }\end{array}$ \\
\hline He et $a^{25}$ & $0.28(0.10-0.77)$ & 9.26 \\
\hline Liao et al ${ }^{30}$ & $0.57(0.31-1.07)$ & 24.70 \\
\hline Wang et al ${ }^{31}$ & $0.32(0.17-0.54)$ & 29.16 \\
\hline Xiong et al ${ }^{34}$ & $0.10(0.01-1.04)$ & 1.87 \\
\hline Yu et a $\left.\right|^{32}$ & $0.41(0.24-0.69)$ & 35.01 \\
\hline $\begin{array}{l}\text { Overall }\left(I^{2}=\mathbf{0 . 0} \%, P=\mathbf{0 . 4 5 2}\right) \\
\text { Test of } E S=1: z=5.97 P=0.000\end{array}$ & $0.39(0.28-0.53)$ & 100 \\
\hline 0.001 & 1.5 & \\
\hline
\end{tabular}

Figure 2 Forest plot for the association between CASC2 expression and OS.

Notes: (A) Unadjusted event rates for OS, (B) adjusted event rates for OS.

Abbreviations: ES, effect size; OS, overall survival; CASC2, cancer susceptibility candidate 2 .

glioma, NSCLC, CRC, ESCC, TC, PC, LAC, PA, CC and $\mathrm{GC}$, when the level of CASC2 expression downregulated in tumor tissue. Meanwhile, patients with higher CASC2 expression often displayed better prognosis and less clinicopathologic features, the same phenomenon were

\section{A}

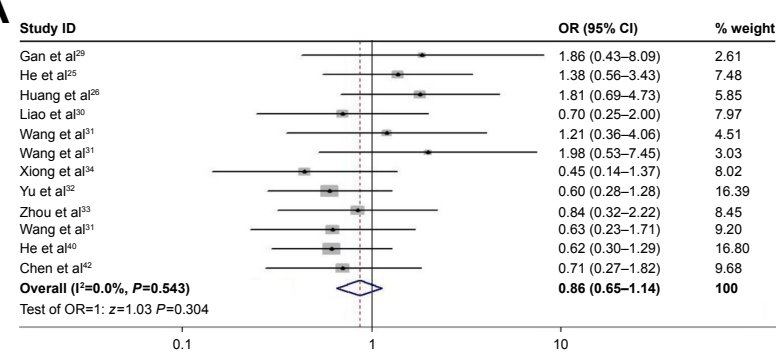

c

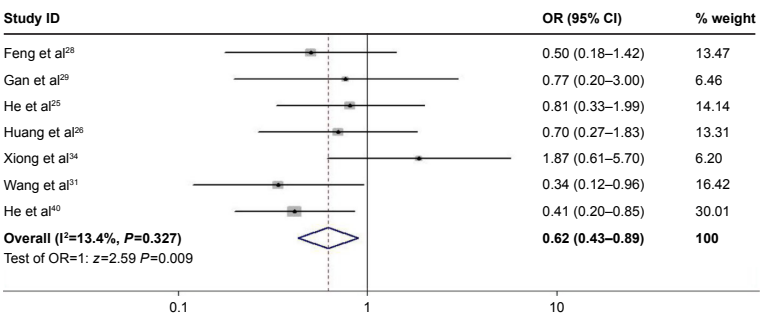

E

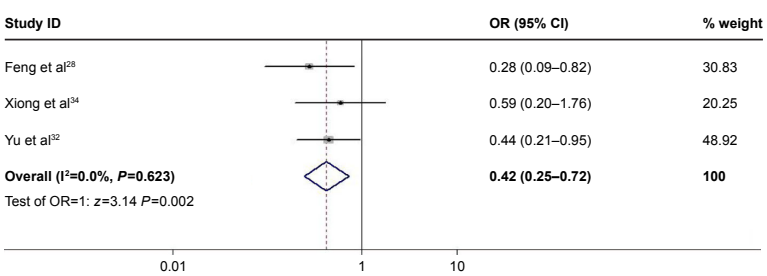

shown in tumor cell lines. Since the lncRNA CASC2 was originally found to be downregulated in endometrial cancer in 2004 as a tumor suppressor, ${ }^{23}$ there have been an increasing number of studies demonstrating that CASC2 expression was related to the prognosis of malignant tumors

B

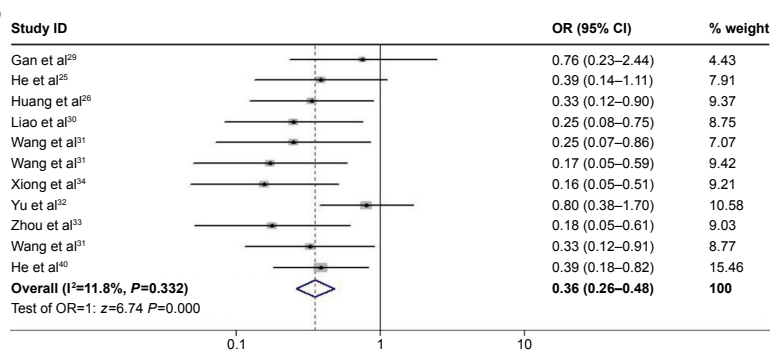

D

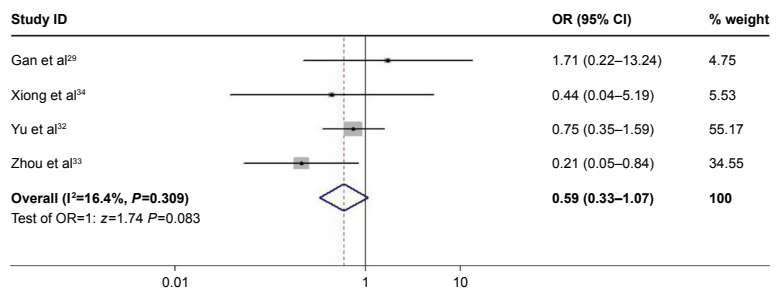

$\mathbf{F}$

\begin{tabular}{|c|c|c|}
\hline Study ID & OR $(95 \% \mathrm{Cl})$ & $\%$ weight \\
\hline Gan et al ${ }^{29}$ & $0.97(0.28-3.30)$ & 10.49 \\
\hline He et a $\left.\right|^{25}$ & $0.53(0.21-1.31)$ & 25.65 \\
\hline Huang et $\mathrm{a}^{26}$ & $0.60(0.19-1.91)$ & 14.96 \\
\hline Zhou et al ${ }^{33}$ & $0.46(0.16-1.31)$ & 21.29 \\
\hline He et al $\left.\right|^{0}$ & $0.79(0.36-1.76)$ & 27.61 \\
\hline $\begin{array}{l}\text { Overall }\left(I^{2}=\mathbf{0 . 0 \%}, P=0.866\right) \\
\text { Test of } \mathrm{OR}=1: z=1.95 \quad P=0.051\end{array}$ & $0.64(0.41-1.00)$ & 100 \\
\hline
\end{tabular}

Figure 3 Forest plot for the association between CASC2 expression and clinicopathological parameters of malignant tumors.

Notes: (A) Correlation between gender and CASC2 expression (female vs male), (B) correlation between TNM and CASC2 expression (stage III-IV vs stage I-II), (C) correlation between LNM and CASC2 expression, (D) correlation between DM and CASC2 expression, (E) correlation between T stage and CASC2 expression, (F) correlation between HD and CASC2 expression.

Abbreviations: LNM, lymph node metastasis; DM, distant metastasis; TNM, tumor node metastasis. 
A Funnel plot with pseudo $95 \%$

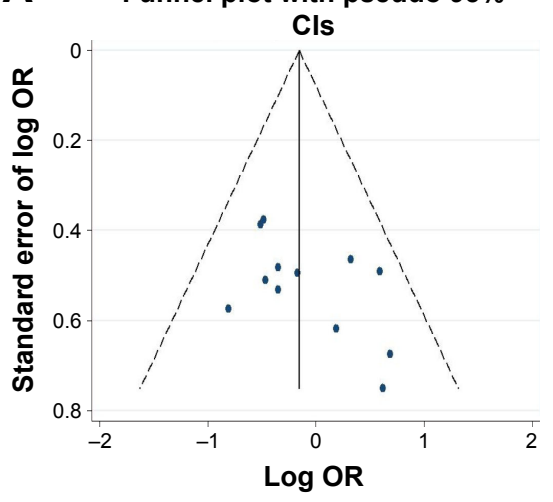

D

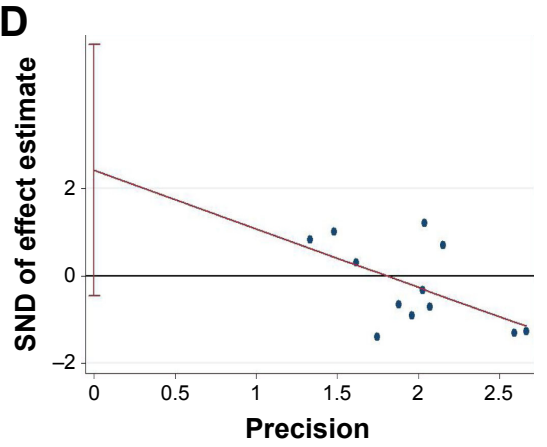

B Funnel plot with pseudo $95 \%$

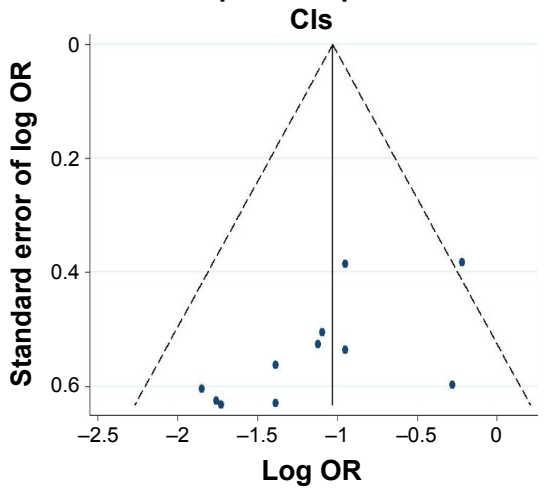

E

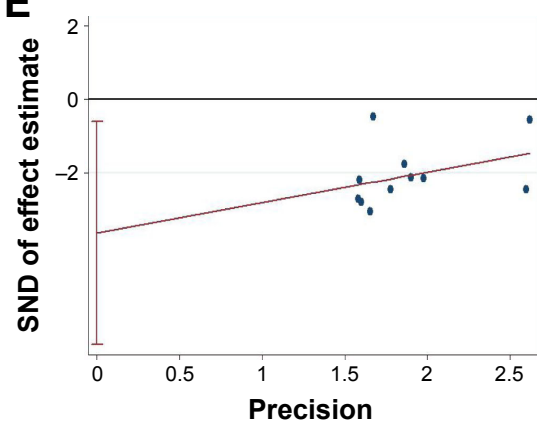

C Funnel plot with pseudo $95 \%$

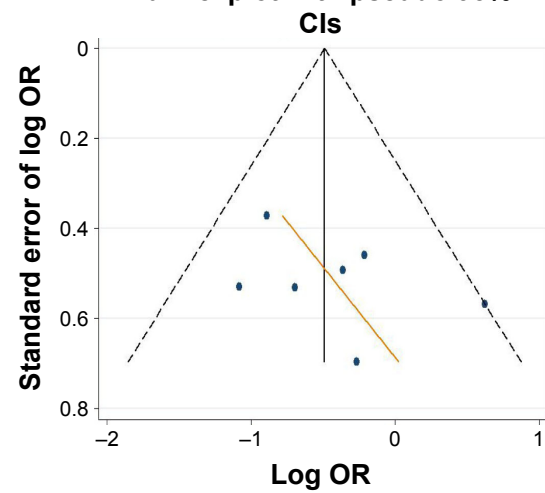

$\mathbf{F}$

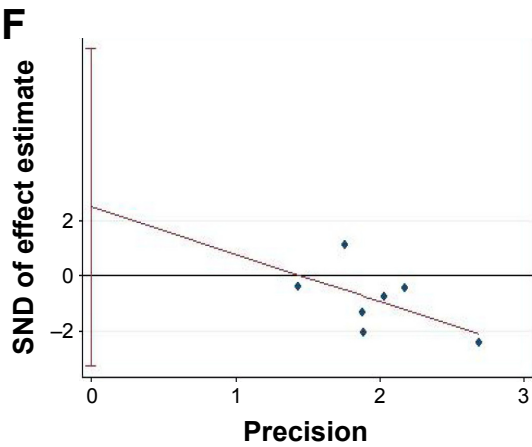

- Study $\longrightarrow$ Regression line $\longmapsto 95 \% \mathrm{Cl}$ for intercept

Figure 4 Funnel plot and Begg's indicator tests of the publication bias.

Notes: (A) Funnel plot for gender, (B) funnel plot for TNM, (C) funnel plot for LNM, (D) Begg's indicator test for gender, (E) Begg's indicator test for TNM, (F) Begg's indicator test for LNM.

Abbreviations: LNM, lymph node metastasis; SND, standard normal deviate; TNM, tumor node metastasis.

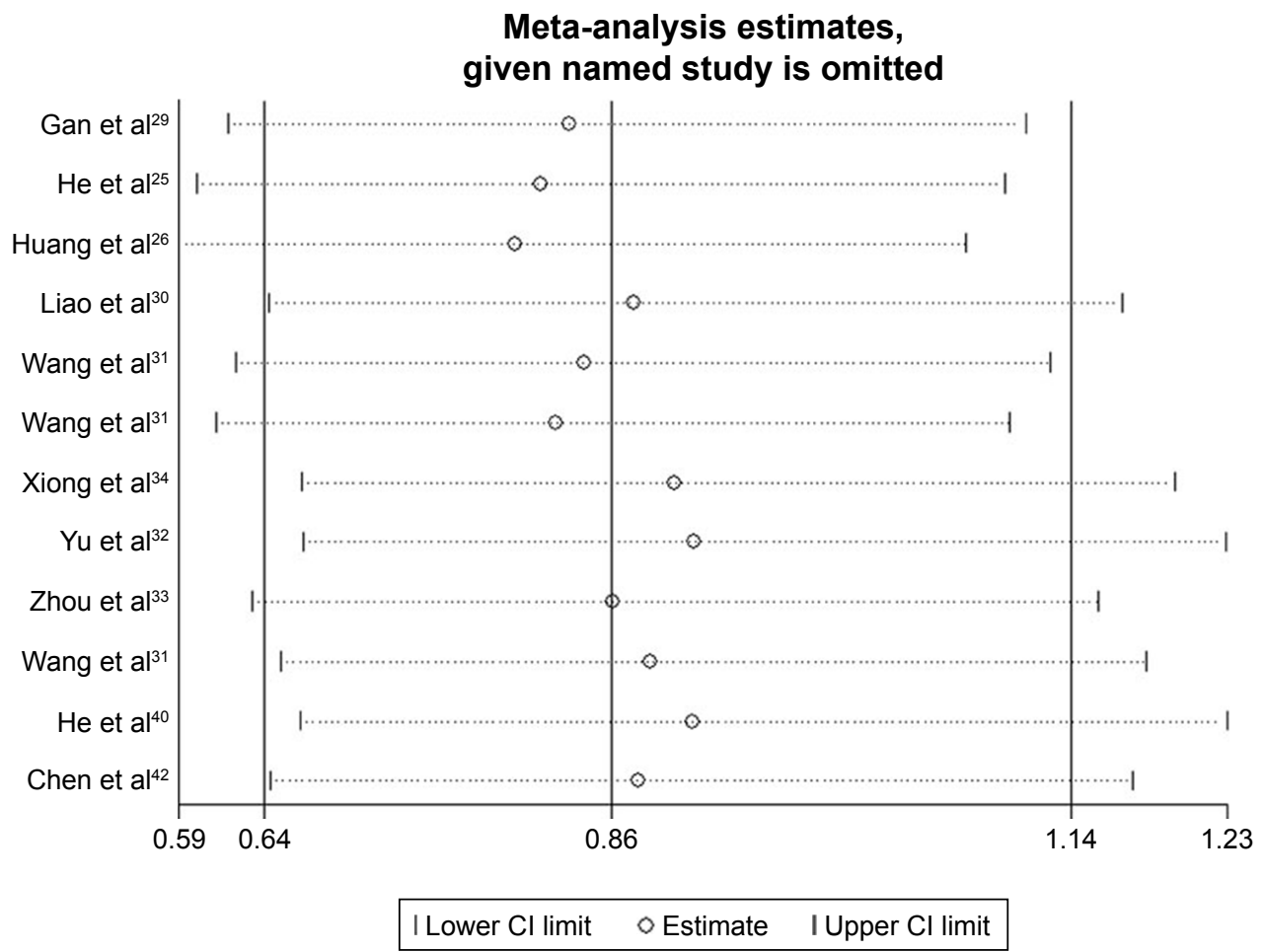

Figure 5 Sensitivity analyses of studies concerning CASC2.

Abbreviation: CASC2, cancer susceptibility candidate 2. 


\begin{tabular}{|c|c|c|c|c|c|c|}
\hline$\underset{\Delta \nabla}{\text { Cancer }}$ & $\begin{array}{c}\text { Coefficient } \\
\Delta \nabla\end{array}$ & $\begin{array}{c}P \text {-value } \\
\Delta \boldsymbol{\nabla}\end{array}$ & $\begin{array}{c}\text { Corrected } \\
\qquad \nabla\end{array}$ & $\underset{\Delta \nabla}{\operatorname{Rank}}$ & $\begin{array}{c}\text { Expression } \\
\Delta \nabla\end{array}$ & $\begin{array}{c}\text { Expression } \\
\Delta \nabla\end{array}$ \\
\hline BLCA & -0.004 & $9.60 \mathrm{e}-01$ & $9.82 e-01$ & 15,920 & 23.08 & 27.71 \\
\hline BRCA & -0.087 & $3.00 \mathrm{e}-01$ & $6.69 \mathrm{e}-01$ & 7,314 & 48.98 & 55.35 \\
\hline CESC & -0.125 & $3.10 \mathrm{e}-01$ & $6.52 \mathrm{e}-01$ & 7,650 & 26.12 & 35.81 \\
\hline COAD & -0.094 & $3.40 \mathrm{e}-01$ & $7.24 \mathrm{e}-01$ & 7,578 & 34.95 & 38.82 \\
\hline ESCA & -0.033 & $7.90 \mathrm{e}-01$ & $9.87 e-01$ & 13,347 & 35.25 & 42.46 \\
\hline GBM & -0.038 & $6.60 \mathrm{e}-01$ & $9.54 \mathrm{e}-01$ & 11,523 & 77.38 & 90.36 \\
\hline HNSC & 0.01 & $8.90 \mathrm{e}-01$ & $9.64 e-01$ & 15,299 & 15.01 & 18.75 \\
\hline KIRC & -0.06 & $4.40 \mathrm{e}-01$ & $5.69 \mathrm{e}-01$ & 12,832 & 60.19 & 67.45 \\
\hline KIRP & -0.25 & $1.00 \mathrm{e}-01$ & $2.33 \mathrm{e}-01$ & 6,934 & 116.2 & 121.43 \\
\hline LAML & -0.082 & $4.30 \mathrm{e}-01$ & $7.71 \mathrm{e}-01$ & 8,396 & 11.65 & 16.29 \\
\hline LGG & -0.034 & $7.10 \mathrm{e}-01$ & $7.81 \mathrm{e}-01$ & 15,242 & 122.12 & 139.94 \\
\hline LIHC & 0.054 & $5.50 e-01$ & $7.69 \mathrm{e}-01$ & 11,258 & 6.37 & 9.57 \\
\hline LUAD & -0.068 & $3.60 \mathrm{e}-01$ & $6.00 \mathrm{e}-01$ & 9,964 & 37.86 & 48.16 \\
\hline LUSC & 0.062 & $3.90 \mathrm{e}-01$ & $8.22 \mathrm{e}-01$ & 7,920 & 24.27 & 29.61 \\
\hline OV & -0.01 & $8.90 \mathrm{e}-01$ & $9.81 e-01$ & 15,228 & 68.99 & 83.88 \\
\hline PAAD & -0.268 & $1.60 \mathrm{e}-02$ & $9.75 e-02$ & 2,765 & 44.57 & 52.83 \\
\hline READ & 0.178 & $3.90 \mathrm{e}-01$ & $9.57 e-01$ & 6,575 & 37.04 & 40.24 \\
\hline SARC & -0.265 & $1.50 \mathrm{e}-02$ & $1.27 \mathrm{e}-01$ & 1,863 & 26.58 & 33.0 \\
\hline SKCM & -0.073 & $2.90 \mathrm{e}-01$ & $4.98 \mathrm{e}-01$ & 9,253 & 13.39 & 17.42 \\
\hline STAD & -0.049 & $5.60 \mathrm{e}-01$ & $8.46 \mathrm{e}-01$ & 11,107 & 49.51 & 58.94 \\
\hline UCEC & 0.011 & $9.20 \mathrm{e}-01$ & $9.95 e-01$ & 15,224 & 64.61 & 80.28 \\
\hline
\end{tabular}

Figure 6 Cox regression results of CASC2 expression in malignant tumors.

Abbreviations: BLAC, Bladder urothelial carcinoma; BRCA, Breast invasive carcinoma; CESC, Cervical and endocervical cancers; COAD, Colon adenocarcinoma; ESCA, Esophageal carcinoma; GBM, Glioblastoma multiforme; HNSC, Head and Neck squamous cell carcinoma; KIRC, kidney renal clear cell carcinoma; KIRP, Kidney renal papillary cell carcinoma; LGG, Brain Lower Grade Glioma; LAML, Acute Myeloid Leukemia; LIHC, Liver hepatocellular carcinoma; LUAD, Lung adenocarcinoma; LUSC, Lung squamous cell carcinoma; PAAD, Pancreatic adenocarcinoma; OV, Ovarian serous cystadenocarcinoma; READ, Rectum adenocarcinoma; SARC, Sarcoma; SKCM, Liver hepatocellular carcinoma; STAD, Stomach adenocarcinoma; UCEC, Uterine Corpus Endometrial Carcinoma.

through different mechanisms. For example, one of the most prominent events was the increased ability of tumor cell proliferation in NSCLC, HCC, CC, CRC, glioma, TC, PC, and $\mathrm{GC}$, where patients presented with low levels of CASC2 expression. ${ }^{25,26,28-35}$ Decreased CASC2 expression in PC, GC, LAC and ESCC was associated with LNM and DM. . $^{32,33,40,41}$ And, in HCC, CRC, glioma, GC and PA, a low level of CASC2 was related to migration and invasion. ${ }^{29,30,31,33,35,42}$ Additionally, CASC2 expression was observed to be significantly correlated with TNM and T stage in NSCLC, CC, TC, PC, GC, LAC and ESCC..$^{25,26,32-34,40,41}$ On the contrary, in HCC, NSCLC, CC, CRC, glioma, TC and PA cells, a higher expression of CASC2 exerted significant tumor suppressing functions through promoting cell apoptosis and arresting cell cycle in vitro, as well as inhibiting tumor cell growth and reducing tumor size..$^{25,26,29-31,34,35,42}$ In the meantime, we further strengthened the results of our meta-analysis by bioinformatics. In the Kaplan-Meier curves with log-rank analysis, we found that the OS of patients with KIRC, LGG, PAAD and SARC was longer compared with those who had lower CASC2 expression. Those results all illustrated that CASC2 might play the role of tumor suppressor in various tumors. A new therapeutic strategy through upregulating the CASC2 expression may benefit cancer patients.

\section{Limitation and strengths}

There were some limitations in this meta-analysis. First, the potential publication bias suggested that the tests were likely to be underpowered. Second, the relatively small number of studies which only came from China limited our ability to identify the relationships between the CASC2 and malignant tumors. The small number of studies also limited the 
A

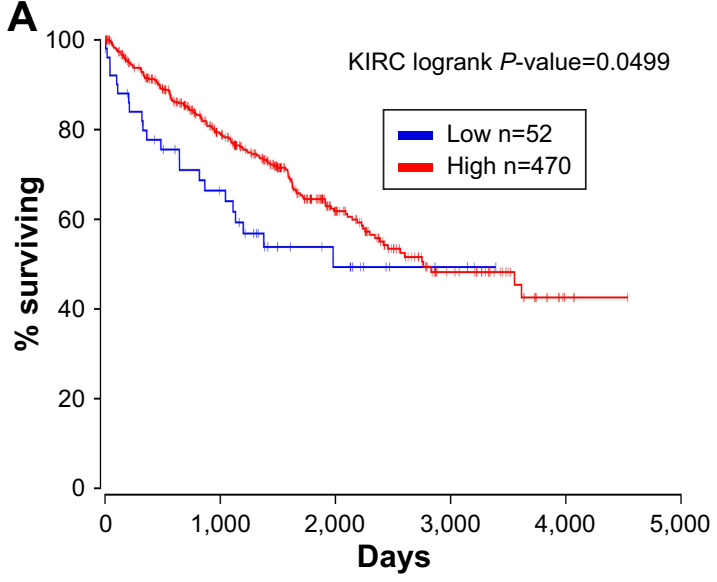

C

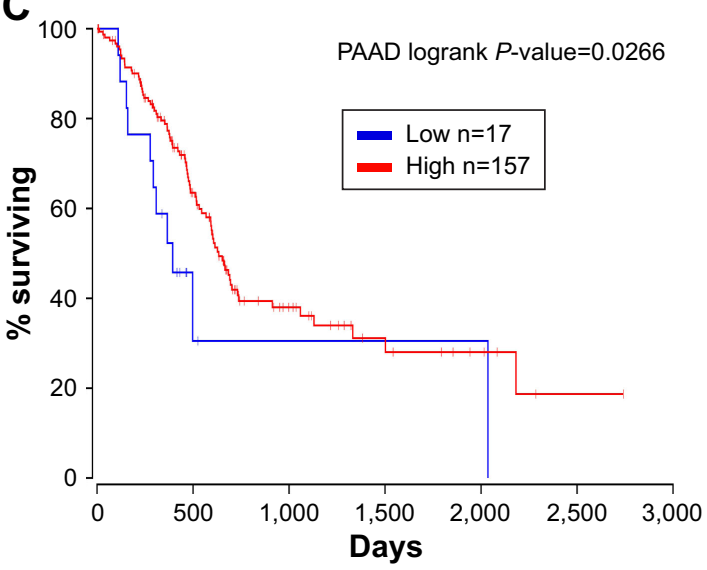

B

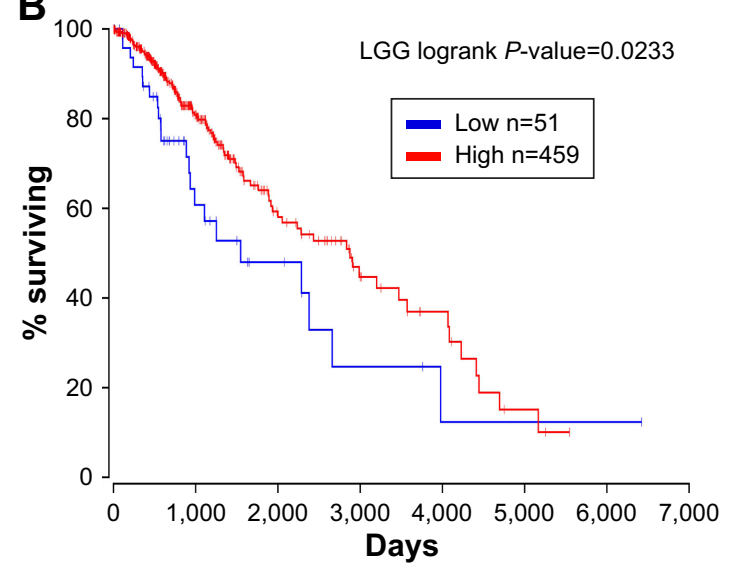

D

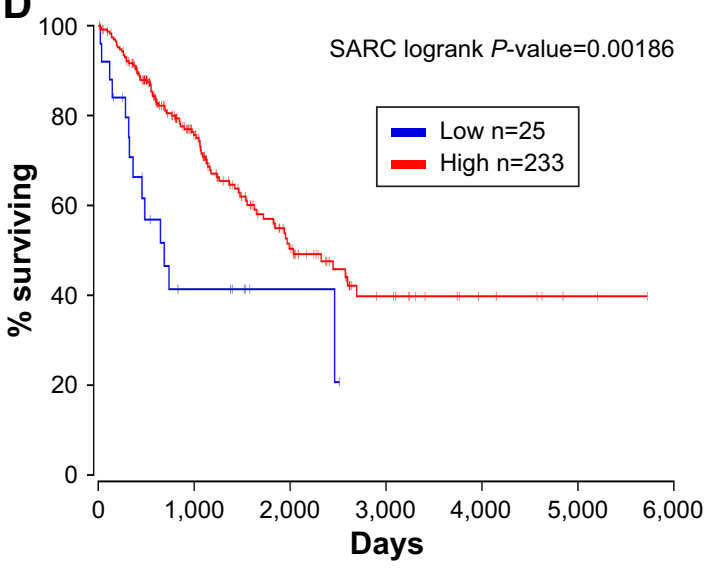

Figure 7 The prognostic significance of CASC2 expression in malignant tumors from TCGA data.

Notes: (A) The survival curve of patients with KIRC, (B) the survival curve of patients with LGG, (C) the survival curve of patients with PAAD, (D) the survival curve of patients with SARC.

Abbreviations: KIRC, kidney renal clear cell carcinoma; LGG, brain lower grade glioma; PAAD, pancreatic adenocarcinoma; SARC, sarcoma; TCGA, The Cancer Genome Atlas.

assessment of heterogeneity related to study quality, and our data may not represent global populations. Third, there is a significant correlation between the status of prognosis and the treatment of patients, and these differences might have a great impact on HR. Finally, unpublished data, non-English language studies, and missed studies may exist and might have influenced our results. Therefore, more studies are needed to make reliable quantitative statements about the relation of CASC2 to malignant tumors. Despite the limitations of the present study, the observed results may be viewed as hypotheses generating and supporting further studies.

\section{Conclusion}

In conclusion, results from this meta-analysis supported a recent and growing body of evidence that the expression of CASC2 is negatively correlated with the prognosis of cancers. Moreover, bioinformatics analysis revealed the correlation between the higher expression of CASC2 and longer OS in patients with malignant tumors. Our findings indicated that CASC2 might be a powerful tool for predicting the progression and prognosis of malignant tumor patients. More studies are needed to determine whether overexpression of CASC2 can benefit patients with various tumors in the future. Future studies focused on CASC2 are also needed to explore mechanisms for tumor initiation and progression.

\section{Acknowledgments}

This work was supported by grants from the National Natural Science Foundation of China (No 81560051, No 81260023, No 81460045 and No 81460010), the "Significant New Drug Creation", Project of National Science and Technology Major Project (No 2014Z × 09303305), the Youth Science Foundation from Science and Technology Department of Jiangxi Province (No 20,171BAB215002), China Postdoctoral Science Foundation funded project (2017 M622107), the Postdoctoral Science Foundation of Jiangxi Province (No 2016KY51) and the Graduate Innovation Fund of Jiangxi Province (No YC2016-B024). 


\section{Author contributions}

LY and SC are co-first authors on this manuscript. All authors contributed toward data analysis, drafting and revising the paper and agree to be accountable for all aspects of the work.

\section{Disclosure}

The authors report no conflicts of interest in this work.

\section{References}

1. Ferlay J, Soerjomataram I, Dikshit R, et al. Cancer incidence and mortality worldwide: sources, methods and major patterns in GLOBOCAN 2012. Int J Cancer. 2015;136(5):E359-E386.

2. Torre LA, Bray F, Siegel RL, et al. Global cancer statistics, 2012. $C A$ Cancer J Clin. 2015;65(2):87-108.

3. Bray F, Ren JS, Masuyer E, Ferlay J. Global estimates of cancer prevalence for 27 sites in the adult population in 2008. Int J Cancer. 2013; 132(5):1133-1145.

4. Zhang H, Lin Y, Li K, et al. Naturally existing oncolytic virus M1 is nonpathogenic for the nonhuman primates after multiple rounds of repeated intravenous injections. Hum Gene Ther. 2016;27(9):700-711.

5. Siegel RL, Miller KD, Jemal A. Cancer statistics 2017. CA Cancer J Clin. 2017;67(1):7-30.

6. Bhaumik P, Gopalakrishnan C, Kamaraj B, Purohit R. Single nucleotide polymorphisms in microRNA binding sites: implications in colorectal cancer. Scientific WorldJournal. 2014;2014:547154.

7. Kumar A, Purohit R. Use of long term molecular dynamics simulation in predicting cancer associated SNPs. PLoS Comput Biol. 2014; 10(4):e1003318.

8. Desantis CE, Lin CC, Mariotto AB, et al. Cancer treatment and survivorship statistics, 2014. CA Cancer J Clin. 2014;64(4):252-271.

9. Ponting CP, Oliver PL, Reik W. Evolution and functions of long noncoding RNAs. Cell. 2009;136(4):629-641.

10. Mercer TR, Dinger ME, Mattick JS. Long non-coding RNAs: insights into functions. Nat Rev Genet. 2009;10(3):155-159.

11. Loewer S, Cabili MN, Guttman M, et al. Large intergenic non-coding RNA-RoR modulates reprogramming of human induced pluripotent stem cells. Nat Genet. 2010;42(12):1113-1117.

12. Sigova AA, Mullen AC, Molinie B, et al. Divergent transcription of long noncoding RNA/mRNA gene pairs in embryonic stem cells. Proc Natl Acad Sci U S A. 2013;110(8):2876-2881.

13. Gupta RA, Shah N, Wang KC, et al. Long non-coding RNA HOTAIR reprograms chromatin state to promote cancer metastasis. Nature. 2010;464(7291):1071-1076.

14. Tsai MC, Spitale RC, Chang HY. Long intergenic noncoding RNAs: new links in cancer progression. Cancer Res. 2011;71(1):3-7.

15. Gutschner T, Hämmerle M, Eissmann M, et al. The noncoding RNA MALAT1 is a critical regulator of the metastasis phenotype of lung cancer cells. Cancer Res. 2013;73(3):1180-1189.

16. Geisler S, Coller J. RNA in unexpected places: long non-coding RNA functions in diverse cellular contexts. Nat Rev Mol Cell Biol. 2013; 14(11):699-712.

17. Djebali S, Davis CA, Merkel A, et al. Landscape of transcription in human cells. Nature. 2012;489(7414):101-108.

18. Martens-Uzunova ES, Böttcher R, Croce CM, et al. Long noncoding RNA in prostate, bladder, and kidney cancer. Eur Urol. 2014;65(6): $1140-1151$.

19. Takahashi K, Yan I, Haga H, Patel T. Long noncoding RNA in liver diseases. Hepatology. 2014;60(2):744-753.

20. Yang X, Xie X, Xiao YF, et al. The emergence of long non-coding RNAs in the tumorigenesis of hepatocellular carcinoma. Cancer Lett. 2015;360(2):119-124.

21. Khachane AN, Harrison PM. Mining mammalian transcript data for functional long non-coding RNAs. PLoS One. 2010;5(4):e10316.
22. Baldinu P, Cossu A, Manca A, et al. Identification of a novel candidate gene, CASC2, in a region of common allelic loss at chromosome 10q26 in human endometrial cancer. Hum Mutat. 2004;23(4):318-326.

23. Baldinu P, Cossu A, Manca A, et al. CASC2a gene is down-regulated in endometrial cancer. Anticancer Res. 2007;27(1A):235-243.

24. Wang $\mathrm{P}$, Liu YH, Yao YL, et al. Long non-coding RNA CASC2 suppresses malignancy in human gliomas by miR-21. Cell Signal. 2015;27(2):275-282.

25. He X, Liu Z, Su J, et al. Low expression of long noncoding RNA CASC2 indicates a poor prognosis and regulates cell proliferation in non-small cell lung cancer. Tumour Biol. 2016;37:9503-9510.

26. Huang $\mathrm{G}, \mathrm{Wu} \mathrm{X}, \mathrm{Li} \mathrm{S}, \mathrm{Xu} X, \mathrm{Zhu} \mathrm{H}$. The long noncoding RNA CASC2 functions as a competing endogenous RNA by sponging miR-18a in colorectal cancer. Sci Rep. 2016;6:26524.

27. Cao Y, Xu R, Xu X, et al. Downregulation of lncRNA CASC2 by microRNA-21 increases the proliferation and migration of renal cell carcinoma cells. Mol Med Rep. 2016;14(1):1019-1025.

28. Feng Y, Zou W, Hu C, Zch W, et al. Modulation of CASC2/miR-21/ PTEN pathway sensitizes cervical cancer to cisplatin. Arch Biochem Biophys. 2017;623-624:20-30.

29. Gan Y, Han N, He X, et al. Long non-coding RNA CASC2 regulates cell biological behaviour through the MAPK signalling pathway in hepatocellular carcinoma. Tumour Biol. 2017;39(6):1393383435.

30. Liao Y, Shen L, Zhao H, et al. LncRNA CASC2 interacts with miR181a to modulate glioma growth and resistance to TMZ through PTEN pathway. J Cell Biochem. 2017;118(7):1889-1899.

31. Wang R, Li Y, Zhu G, et al. Long noncoding RNA CASC2 predicts the prognosis of glioma patients and functions as a suppressor for gliomas by suppressing Wnt/ $\beta$-catenin signaling pathway. Neuropsychiatr Dis Treat. 2017;13:1805-1813.

32. Yu Y, Liang S, Zhou Y, Li S, Li Y, Liao W. HNF1A/CASC2 regulates pancreatic cancer cell proliferation through PTEN/Akt signaling. $J$ Cell Biochem. Epub 2017 Sep 2.

33. Zhou J, Huang H, Tong S, Huo R. Overexpression of long non-coding RNA cancer susceptibility 2 inhibits cell invasion and angiogenesis in gastric cancer. Mol Med Rep. 2017;16(4):5235-5240.

34. Xiong X, Zhu H, Chen X. Low expression of long noncoding RNA CASC2 indicates a poor prognosis and promotes tumorigenesis in thyroid carcinoma. Biomed Pharmacother. 2017;93:391-397.

35. Wang Y, Liu Z, Yao B, et al. Long non-coding RNA CASC2 suppresses epithelial-mesenchymal transition of hepatocellular carcinoma cells through CASC2/miR-367/FBXW7 axis. Mol Cancer. 2017;16(1):123.

36. Guo Q, Zhao Y, Chen J, et al. BRAF-activated long non-coding RNA contributes to colorectal cancer migration by inducing epithelialmesenchymal transition. Oncol Lett. 2014;8(2):869-875.

37. Li L, Zhang L, Zhang Y, Zhou F. Increased expression of LncRNA BANCR is associated with clinical progression and poor prognosis in gastric cancer. Biomed Pharmacother. 2015;72:109-112.

38. Fan YH, Ji CX, Xu B, et al. Long noncoding RNA activated by TGF- $\beta$ in human cancers: a meta-analysis. Clin Chim Acta. 2017;468:10-16.

39. Altman DG, McShane LM, Sauerbrei W, Taube SE. Reporting Recommendations for Tumor Marker Prognostic Studies (REMARK): explanation and elaboration. PLoS Med. 2012;9(5):e1001216.

40. He J, Tian H, Liu K, Yiu J. Long non-coding RNA CASC2 serves as an onco-suppressor in human esophageal squamous cell carcinoma through inhibition of NF- KB pathway. Int J Clin Exp Med. 2017;10(2):2800-2808.

41. Wang D, Gao ZM, Han LG, Xu F, Liu K, Shen Y. Long noncoding RNA CASC2 inhibits metastasis and epithelial to mesenchymal transition of lung adenocarcinoma via suppressing SOX4. Eur Rev Med Pharmacol Sci. 2017;21(20):4584-4590.

42. Chen $\mathrm{S}$, Zhang $\mathrm{S}, \mathrm{Wu} \mathrm{H}$, et al. The long noncoding RNA CASC2 inhibits pituitary adenoma progression by inhibiting HMGA2 expression. Int J Clin Exp Med. 2017;10(10):14458-14467.

43. Zhou S, Wang J, Zhang Z. An emerging understanding of long noncoding RNAs in kidney cancer. J Cancer Res Clin Oncol. 2014; 140(12):1989-1995. 
OncoTargets and Therapy

\section{Publish your work in this journal}

OncoTargets and Therapy is an international, peer-reviewed, open access journal focusing on the pathological basis of all cancers, potential targets for therapy and treatment protocols employed to improve the management of cancer patients. The journal also focuses on the impact of management programs and new therapeutic agents and protocols on

patient perspectives such as quality of life, adherence and satisfaction. The manuscript management system is completely online and includes a very quick and fair peer-review system, which is all easy to use. Visit http://www.dovepress.com/testimonials.php to read real quotes from published authors.

Submit your manuscript here: http://www.dovepress.com/oncotargets-and-therapy-journal 